\title{
Spacers with boluses applied to various sites of oral squamous cell carcinoma: Technical note and retrospective case series
}

\author{
KUNIO YOSHIZAWA ${ }^{1}$, SHINICHI AOKI ${ }^{2}$, KAN MARINO $^{2}$, MASAKI MATSUDA $^{2}$, \\ AKINORI MOROI ${ }^{1}$ and KOICHIRO UEKI ${ }^{1}$ \\ ${ }^{1}$ Department of Oral Maxillofacial Surgery, Division of Medicine, Interdisciplinary Graduate School; \\ ${ }^{2}$ Department of Radiology, School of Medicine, University of Yamanashi, Chuo, Yamanashi 409-3898, Japan
}

Received March 24, 2021; Accepted July 2, 2021

DOI: $10.3892 / \mathrm{mco} .2021 .2349$

\begin{abstract}
The present report describes a case series in which spacers with boluses were used at various sites in the oral cavity to enhance the therapeutic effect of radiation therapy in oral squamous cell carcinoma. In radiotherapy, the surface dose is reduced due to the build-up region of $\mathrm{X}$-rays. In the present study, a bolus was used to complement the build-up region and increase the surface dose effect. A total of 7 patients with oral cancer from a primary care hospital underwent radiation therapy using spacers and added boluses to improve the surface dose effect. The spacer was made from a plastic splint and the bolus was connected to the splint with a quick self-curing resin. There were no complaints of pain or adverse events from the patients while wearing the intraoral splint. A total of 2 of the 7 patients were subsequently confirmed as having progressed disease, and the remaining 5 are currently being managed following a complete response to treatment. The spacers used at various sites of oral squamous cell carcinoma were safe and effective and did not cause any severe adverse effects.
\end{abstract}

\section{Introduction}

Reports show that in Japan, the number of cancer patients is increasing annually (1). Similarly, in the United States, the numbers are also increasing annually and cancer deaths are second only to heart diseases (2). Head and neck cancer, including oral squamous cell carcinoma (OSCC), is the sixth leading malignancy worldwide $(2,3)$ with squamous cell carcinoma accounting for at least $90 \%$ of all oral malignancies (4).

Correspondence to: Dr Kunio Yoshizawa, Department of Oral Maxillofacial Surgery, Division of Medicine, Interdisciplinary Graduate School, University of Yamanashi, 1110 Shimokato, Chuo, Yamanashi 409-3898, Japan

E-mail: yoshizawak@yamanashi.ac.jp

Key words: bolus, radiotherapy, oral squamous cell carcinoma, spacer
Radiotherapy is an important treatment modality for OSCC because of its relatively high radio-sensitivity and since the maintenance of oral function and morphology is very important for maintaining the patients' quality of life and activities of daily living (5).

Radiotherapy should be administered carefully in order to administer high doses to only the target area and to not administer excess radiation to other normal tissues. In order to avoid osteoradionecrosis (ORN), it has been found to be useful to place spacers to increase the distance between the mandible and the irradiation site of the tongue carcinoma during radiotherapy for interstitial brachytherapy $(6,7)$. In prostate cancer, while using external beam radiation therapy, the insertion of a hydrogel into the prostate-rectal interface to reduce significant exposure to the rectum has been shown to be effective in preventing radiation-related complications $(8,9)$. Similarly, while using external irradiation for oral cancer, it has been considered effective to wear a spacer to maintain the distance between the adjacent normal tissue and the irradiation site of the lesion. X-rays used during external radiation therapy are characterised by the maximum absorbed dose when they enter the body within an area of about $10 \mathrm{~mm}$ from the body surface (10). Since oral cancer is a lesion on the surface of the body, a bolus, which has almost the same characteristics as the human body, can be placed over the lesion to maximize the dose at the surface layer of the lesion by utilising the build-up effect (11). Based on the results of previous studies (10-13), if bolus build-up areas are not created, there may be dose reductions near the surface and sufficient therapeutic effects may not be achieved.

Taking into account the extensive losses of form and function caused by resections, radiation therapy is particularly advantageous in cases of extensive superficial oral cancers. High energy X-rays of 4-6 MVX used for treating oral cancer have good linearity and can easily irradiate evenly to the edge of the irradiation field; however, the surface dose effect is low due to the build-up effect (14). Since oral cancer originates from the superficial mucosa and becomes malignant, external radiation therapy must increase the surface dose effect and cure the primary site $(12,14)$.

To enhance the surface dose effect of external radiotherapy, we developed a spacer with bolus material to adhere to the primary site and used it in patients undergoing 
radiotherapy. The bolus material is a water-equivalent material $(11,12)$ which can be adhered to and placed according to the contour shape of the oral mucosa to complement the build-up area. Commercially available bolus materials are about $5-10 \mathrm{~mm}$ thick, and by placing them on the spacer, a large and uniform dose distribution on the mucosal surface can be achieved. The current National Comprehensive Cancer Network Breast guidelines state: 'Special consideration should be given to the use of bolus material to ensure that the skin dose is adequate' $(15,16)$. Worldwide, bolus material has been used frequently in breast cancer treatment to increase the surface dose effect (17); however, to the best of our knowledge, there have been only two reports of its use in oral cancer $(18,19)$. In these case reports, the indications were limited to tongue cancer and cancer of the palate; however, in our case reports, we used their method at various sites of oral cancer and reported on the clinical courses and adverse effects such as oral mucositis, dysgeusia, and ORN.

The main purpose of the spacer is to improve the accuracy of positioning of the irradiation site during radiotherapy and to prevent excessive radiation to the surrounding normal tissues, thereby preventing ORN and severe mucositis (6,20-22).

This study described a case series in which spacers with boluses were used at various sites in the oral cavity to enhance the therapeutic effect of radiation therapy in oral squamous cell carcinoma.

\section{Patients and methods}

Patients. We conducted a prospective observational study among oral cancer patients aged $>18$ years who underwent adequate medical follow-up from October 2019 and January 2021 in Yamanashi University Hospital. This study was performed in accordance with the Declaration of Helsinki with the approval of the Ethics Committee of Yamanashi University Hospital (no. 2352). According to the guidelines of the Ethics Committee, all patient data were anonymised before use and written informed consent was obtained from each patient and/or family before inclusion in the study. Patients were free to withdraw from the study at any time. The inclusion criteria for this research were as follows: Adults $>20$ years of age, diagnoses of oral squamous cell carcinoma, and no recent rapid exacerbations. The exclusion criteria were as follows: Having any acute or chronic condition that would limit the ability of the patient to participate in the study and refusal by patient and/or family to provide informed consent. There were no exclusions among the enrolled patients.

Five men and two women with oral cancer with an average age of 71.1 years (range, 47-92 years) were included in this study and they each underwent external beam radiation using a spacer with a bolus. Table I shows the characteristics and treatment of the seven cases including the underlying diseases and performance statuses of the ECOGs (23). Table II shows the adverse events according to the Common Terminology Criteria for Adverse Events (CTCAE) guidelines, version 5.0 (24) after external radiotherapy and the histories of tobacco use and the alcohol intake amounts before treatment. In this study, we observed whether spacers with boluses placed on various sites of the oral cavity would induce to intensify local adverse events such as oral mucositis, dysgeusia, and ORN.

Biological effective dose. The following formula were used for the calculation of biological effective dose (BED) (7,25-27).

$\mathrm{BED}=\mathrm{n} \times \mathrm{d} \times(1+\mathrm{d} /[\alpha / \beta])$

$\mathrm{n}$ : Number of times of radiation therapy

$\mathrm{d}$ : Dose of radiation per one time $(\mathrm{Gy})$

$\beta / \alpha$ : Factor of recovery on targeted tissue (In oral cancer, this value can be approximated as 10).

In Case 1, BED=7 (Gy) x 5x1.7=59.5 (Gy) was calculated.

In Case 2-7, BED=2 (Gy) x 35x1.2=84.0 (Gy) was calculated.

Making the spacer with a bolus. An impression of the upper and lower jaw was made using irreversible hydrocolloid material (Algiace; Sankin Co.). A cast of the impression was subsequently generated using hard plaster (Newplastone; GC Co.). A 1.5-mm thick plastic disk (Erkodur; Erkodent Co.) was pressed onto the replicated plaster cast of the jaw using a thermoplastic former (Erkopress; Erkodent Co.). Once the curing was completed, the plastic disk was cut-off from the plaster cast, and the rough edges were smoothed using a polisher. A quick self-curing resin (Ortho Crystal; Nissin Dental Inc.) was added to the spacer to enable the bolus with a mass density of $1.03 \mathrm{~g} / \mathrm{cm}^{3}$ (Bolus; Toyo Medic Co.) to conform and adhere to the irradiated area (Figs. 1 and S1). The thickness of the spacer, which was the sum of the plastic base and bolus, was adjusted to obtain a final thickness of $\sim 10 \mathrm{~mm}$ at the equivalent radiation therapy site. Before radiotherapy began, the spacer was set into the patient's mouth, and we confirmed that it was painless to wear and that the lesion could be reproducibly covered with the bolus. Three-dimensional treatment planning was performed by a radiologist with the spacer in the patient's mouth.

\section{Results}

Patient characteristics. Table I shows the characteristics, treatment and its effect in each patient case. In all cases, smoking cessation was successfully achieved, and consuming alcohol was controlled to sobriety during/after treatment. The overall response rate was $71.4 \%$, with five cases showing a complete response (CR) and two cases with progressed disease (PD). Furthermore, as shown in Table II, osteonecrosis of the jaw as an adverse event was 'None' in each of the four cases, grade 1 in two cases, grade 2 in one case, and grade 2 or less in all the cases. Fig. 2 shows the distribution of radiation doses imaged during the radiation therapy planning when the spacer was placed in the oral cavity. The bolus aligned with the lesion, was drawn in gray and was easily identifiable because the absorbed dose was comparable to that of water and lower than the absorbed dose of surrounding tissue.

Clinical course of external radiation. Except for Case 1, external radiation therapy with $4 \mathrm{mV}$ X-rays consisted of 60-70 Gy/30-35 f, with a daily fraction size of 2 Gy and five fractions per week. Case 1 was that of an elderly patient with severe dementia, and the patient and his family refused hospitalisation. Therefore, we decided to use a high dose of 


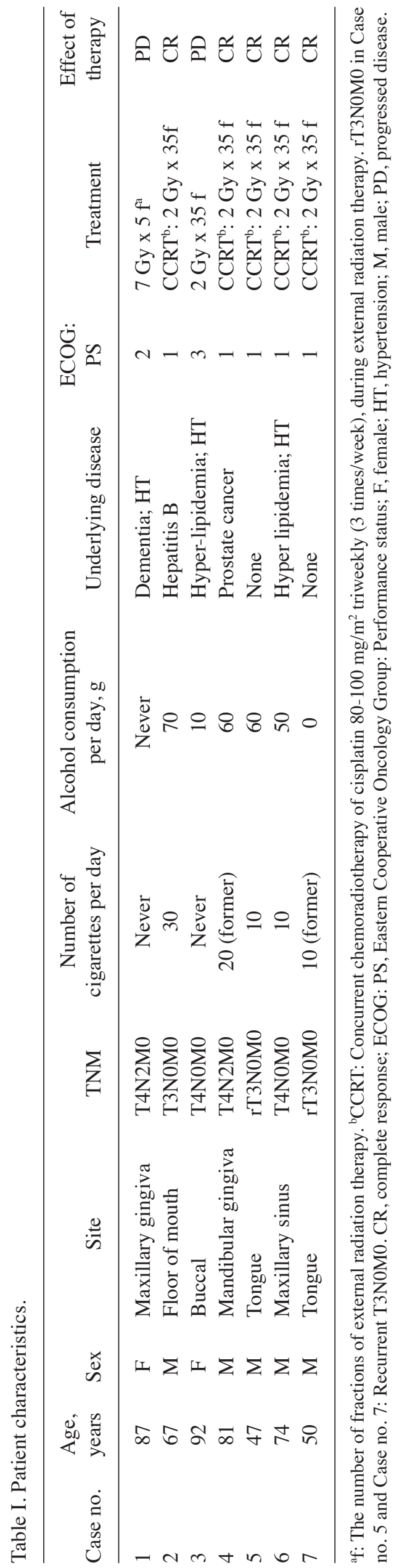

7 Gy per fraction with $6 \mathrm{mV} \mathrm{X-rays} \mathrm{and} \mathrm{provide} \mathrm{outpatient}$ treatment with five radiation treatments.

One year after the completion of treatment, the patient developed recurrent symptoms, was found to have PD and died 6 months later. The other PD patient (Case 3), who was 92 years old, was not selected for combination chemotherapy due to her poor general condition. Six months after the completion of treatment, she developed recurrent symptoms, her general condition deteriorated, and she died. Each of the six patients with CR were in a good general condition and could be treated with concurrent cisplatin chemotherapy, which is considered to be the standard care.

\section{Discussion}

In this study, a conventional spacer was attached to keep the normal tissue away from the irradiation site, and bolus material was attached to the spacer to make it about $10 \mathrm{~mm}$ thick to enhance the surface dose effect at the target site.

The installation of the bolus material on the spacer provided the following two advantages. First, since the bolus material is a water-equivalent, gel-like material, it can be safely fixed close to the lesion site and can assist in identifying the area to be irradiated because it has a different absorbed dose from other areas considered during radiotherapy planning. Second, it increases the surface dose effect near the horizontal margins of the oral squamous cell carcinoma, which makes it effective in preventing recurrences in the same area. In other words, this study was unique in two aspects: The easy identification of the target lesion using $\mathrm{CT}$ with the bolus application facilitating accurate radiotherapy planning and the enhancement of the surface dose effect.

In contrast, since radiotherapy alone may not have had an adequate anti-tumour effect, the combined use of cisplatin is recommended as a curative treatment. In the two cases (Case 1 and Case 3) of recurrence in this study, the intensities of treatment may have been too weak because radiotherapy alone was chosen even though the patients had advanced cancer with invasions of the jawbone. The present study suggested that spacers should be used in combination with cisplatin for the treatment of aggressive oral cancer. Furthermore, in Case 1, the treatment effect may have been insufficient because the irradiation dose was $59.5 \mathrm{~Gy}$ (BED equivalent), which is considered somewhat low for a curative dose due to the choice of small fractional irradiation.

Limitations of the present study were the small number of cases and the short follow-up periods. Since there is a possibility that ORN may occur later than several years, it is necessary to prevent ORN by maintaining thorough oral hygiene during the follow-ups. It is recommended that future studies increase the number of cases and further analyse the treatment effect in prospective multicentre studies to confirm the superiority of the spacer with bolus.

In conclusion, we showed that spacers with boluses can be placed at various sites of the oral cavity and that the osteonecrosis of the jaw as an adverse event was less than grade 2 . This treatment method requires collaboration between dental surgeons and radiologists and may lead to a reduction in adverse events associated with radiotherapy. 
Table II. Investigation of smoking and alcohol consumption before treatment and adverse events of CTCAE ver. 5.0 after external radiotherapy.

\begin{tabular}{lccccc}
\hline Case no. & Age, years & Sex & Mucositis & Dysgeusia & Osteonecrosis of the jaw \\
\hline 1 & 87 & F & 2 & 1 & 1 \\
2 & 67 & M & 2 & 2 & None \\
3 & 92 & F & 3 & 2 & 2 \\
4 & 81 & M & 2 & 2 & None \\
5 & 47 & M & 2 & 2 & None \\
6 & 74 & M & 2 & 1 & None \\
7 & 50 & M & 2 & 2 & 1 \\
\hline
\end{tabular}

${ }^{a}$ CTCAE ver5.0: Common terminology criteria for adverse events version 5.0. F, female; M, male.

A

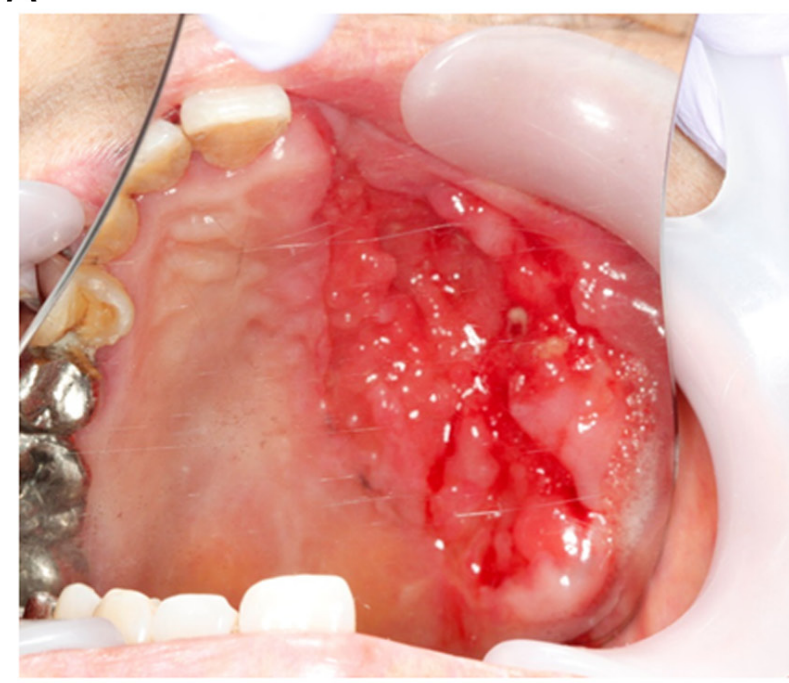

B

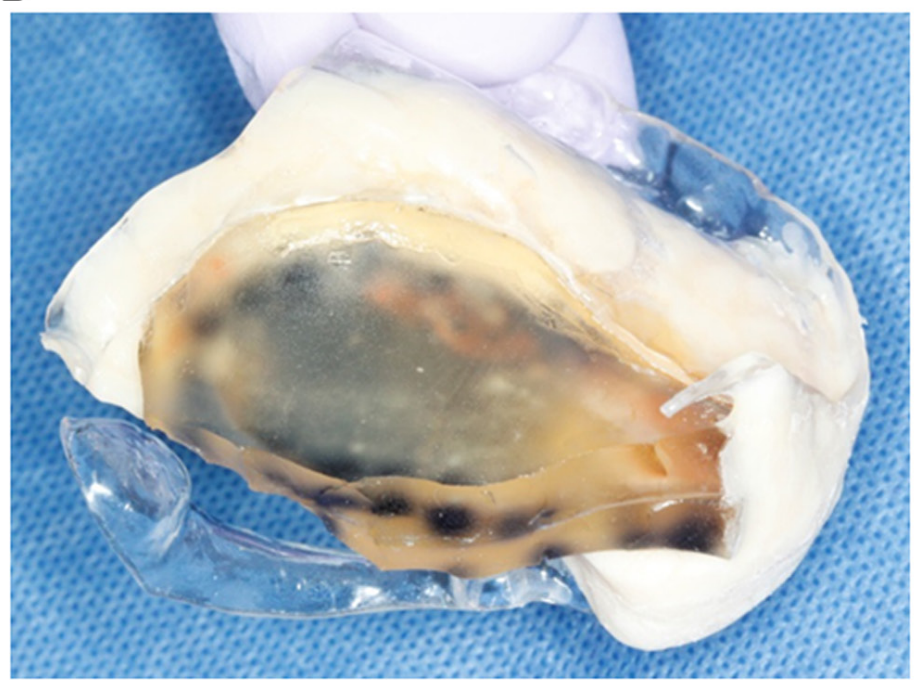

Figure 1. Spacer with bolus in Case 1. (A) A mirror image of the right side maxillary gingival carcinoma. (B) A spacer with bolus material adheres to the irradiated area of the maxillary gingival carcinoma.

A

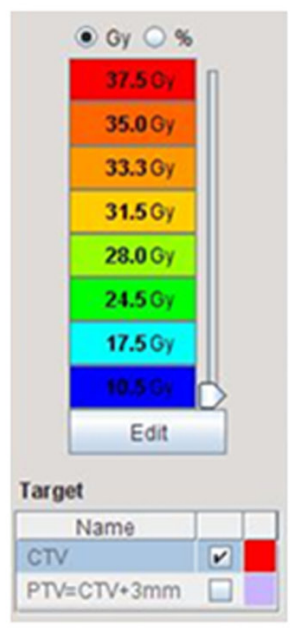

Transverse

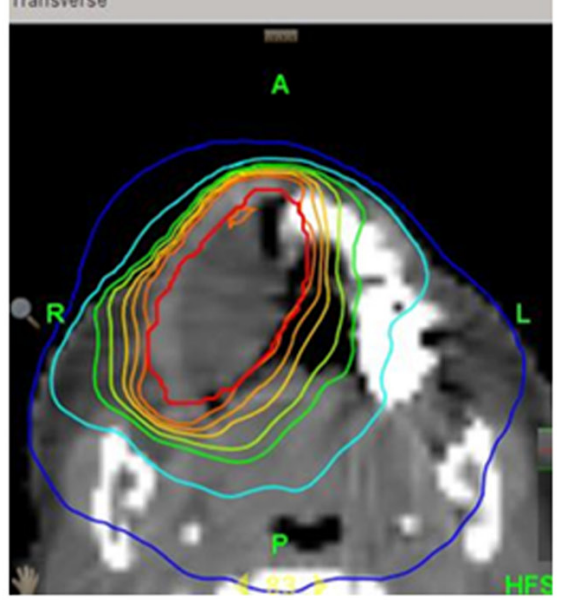

B

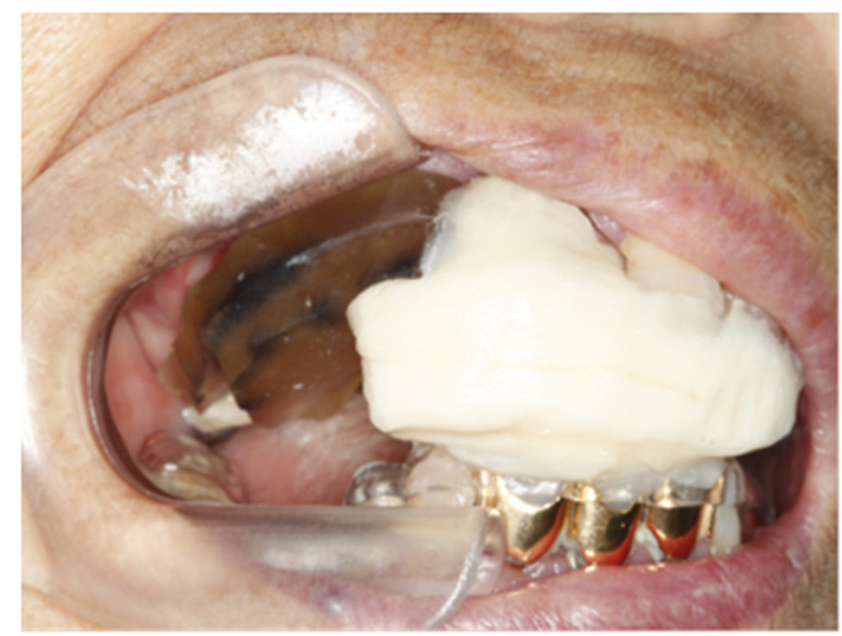

Figure 2. Simulated irradiation dose distribution of Case 1 with spacer including bolus. (A) The area surrounded by the red line indicates the lesion site where the highest irradiation dose was set, and it coincided with the bolus site in the gray area, which was closely aligned with the lesion. (B) Spacer with bolus is shown mounted in the mouth. 


\section{Acknowledgements}

The authors would like to thank Professor Hiroshi Onishi (Department of Radiology, School of Medicine, University of Yamanashi, Chuo, Japan), for his valuable advice for radiotherapy in clinical conference for each case.

\section{Funding}

No funding was received.

\section{Availability of data and materials}

The datasets used and/or analysed during the current study are available from the corresponding author on reasonable request.

\section{Authors' contributions}

KY, KM, SA and MM conceived and designed the study, and conducted data collection. KY, AM and KU researched the literature, and performed the analysis of the data. KM, SA, $\mathrm{MM}, \mathrm{AM}$ and $\mathrm{KU}$ confirmed the authenticity of all the raw data. All authors contributed to the drafting of the manuscript and critically revised the manuscript. All authors read and approved the final manuscript.

\section{Ethics approval and consent to participate}

The present study was approved by the Ethics Committee of Yamanashi University (approval no. 2352; Chuo, Yamanashi, Japan). The present study was conducted in accordance with the Declaration of Helsinki. According to the guidelines of the Ethics Committee, all the patient data were anonymised before use. Written informed consent was obtained from each patient and/or the family before inclusion in the study.

\section{Patient consent for publication}

Written informed consent for publication of the oral photographs in Figs. 1 and 2 and S1 was obtained from each patient or the family.

\section{Competing interests}

The authors declare that they have no competing interests.

\section{References}

1. Ministry of Health Labour and Welfare: Ministry of Health, Labour and Welfare (In Japanese). https://www.mhlw.go.jp/ bunya/kenkou/gan_toukei.html. Accessed January 13, 2021.

2. Siegel RL, Miller KD and Jemal A: Cancer statistics, 2020. CA Cancer J Clin 70: 7-30, 2020.

3. Torre LA, Bray F, Siegel RL, Ferlay J, Lortet-Tieulent J and Jemal A: Global cancer statistics, 2012. CA Cancer J Clin 65: 87-108, 2015

4. Massano J, Regateiro FS, Januário G and Ferreira A: Oral squamous cell carcinoma: Review of prognostic and predictive factors. Oral Surg Oral Med Oral Pathol Oral Radiol Endod 102: 67-76, 2006.

5. Lin A: Radiation therapy for oral cavity and oropharyngeal cancers. Dent Clin North Am 62: 99-109, 2018.
6. Yuasa K, Kawazu T, Morita M, Uehara S, Kunitake N and Kanda S: A new, simple method of making a spacer in interstitial brachytherapy for mobile tongue cancer. Oral Surg Oral Med Oral Pathol Oral Radiol Endod 89: 519-521, 2000.

7. Miura M, Takeda M, Sasaki T, Inoue T, Nakayama T, Fukuda H, Hoshi A, Hoshina M and Shibuya H: Factors affecting mandibular complications in low dose rate brachytherapy for oral tongue carcinoma with special reference to spacer. Int J Radiat Oncol Biol Phys 41: 763-770, 1998.

8. Hamstra DA, Mariados N, Sylvester J, Shah D, Karsh L, Hudes R, Beyer D, Kurtzman S, Bogart J, His RA, et al: Continued benefit to rectal separation for prostate radiation therapy: Final results of a phase III trial. Int J Radiat Oncol Biol Phys 97: 976-985, 2017.

9. Susil RC, McNutt TR, DeWeese TL and Song D: Effects of prostate-rectum separation on rectal dose from external beam radiotherapy. Int J Radiat Oncol Biol Phys 76: 1251-1258, 2010.

10. McKenna MG, Chen XG, Altschuler MD and Bloch P: Calculation of the dose in the build-up region for high energy photon beam. Treatment planning when beam spoilers are employed. Radiother Oncol 34: 63-68, 1995.

11. Sroka M, Reguła J and Lobodziec W: The influence of the bolus-surface distance on the dose distribution in the build-up region. Rep Pract Oncol Radiother 15: 161-164, 2010.

12. Günhan B, Kemikler G and Koca A: Determination of surface dose and the effect of bolus to surface dose in electron Beams. Med Dosim 28: 193-198, 2003.

13. Chu JC, Coia LR, Aziz D and Stafford PM: Dose to superficial node for patients with head and neck cancer treated with $6 \mathrm{MV}$ and 60Co photons. Radiother Oncol 21: 257-260, 1991.

14. Kudchadker RJ, Antolak JA, Morrison WH, Wong PF and Hogstrom KR: Utilization of custom electron bolus in head and neck radiotherapy. J Appl Clin Med Physics 4: 321-333, 2003.

15. Gradishar WJ, Anderson BO, Abraham J, Aft R, Agnese D, Allison KH, Blair SL, Burstein HJ, Dang C, Elias AD, et al: Breast cancer, version 3.2020, NCCN clinical practice guidelines in oncology. J Natl Compr Canc Netw 18: 452-478, 2020.

16. Recht A, Edge SB, Solin LJ, Robinson DS, Estabrook A, Fine RE, Fleming GF, Formenti S, Hudis C, Kirshner JJ, et al: Postmastectomy radiotherapy: Clinical practice guidelines of the American Society of Clinical Oncology. J Clin Oncol 19: 1539-1569, 2001.

17. Vu TT, Pignol JP, Rakovitch E, Spayne J and Paszat L: Variability in radiation oncologists' opinion on the indication of a bolus in post-mastectomy radiotherapy: An international survey. Clin Oncol (R Coll Radiol) 19: 115-119, 2007.

18. Baek S, Ahn S, Ju E and Jung NH: Customized 3D bolus applied to the oral cavity and supraclavicular area for head and neck cancer. In Vivo 35: 579-584, 2021.

19. Nam KY: Radiation prosthetic stents applied to oral cancer patients during the radiation therapy: Case reports. J Dental Rehabil Appl Sci 36: 282-288, 2020 (In Korean).

20. Bedwinek JM, Shukovsky LJ, Fletcher GH and Daley TE: Osteonecrosis in patients treated with definitive radiotherapy for squamous cell carcinomas of the oral cavity and Naso-and oropharynx. Radiology 119: 665-667, 1976.

21. Curi MM and Dib LL: Osteoradionecrosis of the jaws: A retrospective study of the background factors and treatment in 104 cases. J Oral Maxillofac Surg 55: 540-546, 1997.

22. Obinata K, Ohmori K, Tuchiya K, Nishioka T, Shirato H and Nakamura M: Clinical study of a spacer to help prevent osteoradionecrosis resulting from brachytherapy for tongue cancer. Oral Surg Oral Med Oral Pathol Oral Radiol Endod 95: 246-250, 2003.

23. Oken MM, Creech RH, Tormey DC, Horton J, Davis TE, McFadden ET and Carbone PP: Toxicity and response criteria of the Eastern Cooperative Oncology Group. Am J Clin Oncol 5: 649-655, 1982

24. National Cancer Institute: Common Terminology Criteria for Adverse Events (CTCAE) guidelines, version 5.0. 2017. https://ctep.cancer.gov/protocoldevelopment/electronic applications/docs/CTCAE_v5_Quick_Reference_8.5x11.pdf 2017 . Accessed January 14, 2021 .

25. Dale RG: The application of the linear-quadratic dose-effect equation to fractionated and protracted radiotherapy. Br J Radiol 58: 515-528, 1985.

26. Bentzen SM, Dörr W, Gahbauer R, Howell RW, Joiner MC, Jones B, Jones DT, van der Kogel AJ, Wambersie A and Whitmore G: Bioeffect modeling and equieffective dose concepts in radiation oncology-terminology, quantities and units. Radiother Oncol 105: 266-268, 2012.

27. Dutreix J: Expression of the dose rate effect in clinical curietherapy. Radiother Oncol 15: 25-37, 1989. 